\title{
Sparse Partial Equilibrium Tables in Chemically Resolved Reactive Flow
}

\author{
P. Vitello, L.E. Fried, B. Pudliner, T. McAbee
}

This article was submitted to American Physical Society Topical Conference on Shock Compression of Condensed Matter Portland, OR

July 20-25, 2003

\section{July 14, 2003}

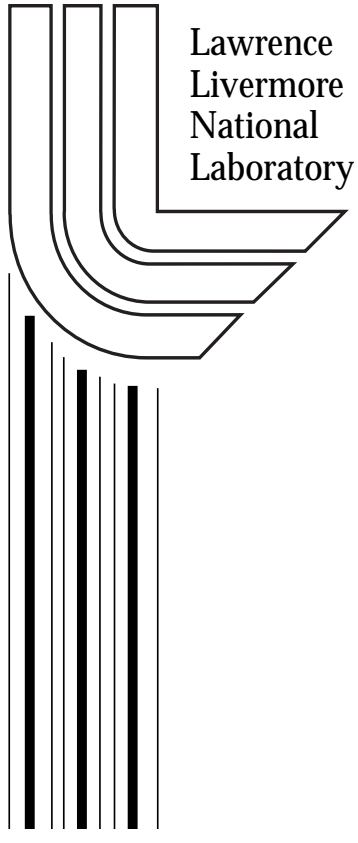




\section{DISCLAIMER}

This document was prepared as an account of work sponsored by an agency of the United States Government. Neither the United States Government nor the University of California nor any of their employees, makes any warranty, express or implied, or assumes any legal liability or responsibility for the accuracy, completeness, or usefulness of any information, apparatus, product, or process disclosed, or represents that its use would not infringe privately owned rights. Reference herein to any specific commercial product, process, or service by trade name, trademark, manufacturer, or otherwise, does not necessarily constitute or imply its endorsement, recommendation, or favoring by the United States Government or the University of California. The views and opinions of authors expressed herein do not necessarily state or reflect those of the United States Government or the University of California, and shall not be used for advertising or product endorsement purposes.

This is a preprint of a paper intended for publication in a journal or proceedings. Since changes may be made before publication, this preprint is made available with the understanding that it will not be cited or reproduced without the permission of the author.

This report has been reproduced directly from the best available copy.

Available electronically at http://www.doc.gov/bridge

Available for a processing fee to U.S. Department of Energy

And its contractors in paper from

U.S. Department of Energy

Office of Scientific and Technical Information

P.O. Box 62

Oak Ridge, TN 37831-0062

Telephone: (865) 576-8401

Facsimile: (865) 576-5728

E-mail: reports@adonis.osti.gov

Available for the sale to the public from

U.S. Department of Commerce

National Technical Information Service

5285 Port Royal Road

Springfield, VA 22161

Telephone: (800) 553-6847

Facsimile: (703) 605-6900

E-mail: orders@ntis.fedworld.gov

Online ordering: http://www.ntis.gov/ordering.htm

OR

Lawrence Livermore National Laboratory

Technical Information Department's Digital Library

http://www.llnl.gov/tid/Library.html 


\title{
Sparse Partial Equilibrium Tables in Chemically Resolved Reactive Flow
}

\author{
Peter Vitello, Laurence E. Fried, Brian Pudliner, and Tom McAbee
}

Lawrence Livermore National Laboratory, Livermore CA 94551

\begin{abstract}
The detonation of an energetic material is the result of a complex interaction between kinetic chemical reactions and hydrodynamics. Unfortunately, little is known concerning the detailed chemical kinetics of detonations in energetic materials. CHEETAH uses rate laws to treat species with the slowest chemical reactions, while assuming other chemical species are in equilibrium. CHEETAH supports a wide range of elements and condensed detonation products and can also be applied to gas detonations. A sparse hash table of equation of state values, called the "cache" is used in CHEETAH to enhance the efficiency of kinetic reaction calculations. For large-scale parallel hydrodynamic calculations, CHEETAH uses MPI communication to updates to the cache. We present here details of the sparse caching model used in the CHEETAH. To demonstrate the efficiency of modeling using a sparse cache model we consider detonations in energetic materials.
\end{abstract}

\section{INTRODUCTION}

Detonation of an energetic material is a complex process involving coupled chemical kinetics and hydrodynamics. While the detailed chemical kinetics of detonations in gases have been extensively studied, chemical kinetic processes governing condensed energetic materials is much less understood. To aid in studying the kinetics of energetic material detonations the CHEETAH [1] thermochemical code was coupled to a multidimensional Arbitrary Lagrangian Eulerian (ALE) hydrocode. The CHEETAH code is used to determine the properties of the reacting energetic materials. Code. CHEETAH allows for products to be in thermochemical equilibrium with an arbitrary number of reactants coupled through rate laws. CHEETAH calculates the chemical composition of the reacting high explosive gases using a high-pressure fluid equation of state (EOS). The transformation of the high explosives into a reacting fluid of small product molecules is based on simplified chemical kinetic schemes, which are determined from measured detonation velocities as a function of size.

\section{CHEETAH}

The EOS for the products is based on an exponential-6 potential [2] for supercritical fluids, with a Murnaghan form [3] for solids and liquids. For the exponential-6 EOS, the parameters are chosen to reproduce shock Hugoniot and static compression data for a wide range of supercritical fluids [4]. This produces an accurate and complete EOS of the exponential-6 fluid based on HMSA integral equation theory and Monte Carlo calculations. Unlike previous detonation product sets, this model is not fit to detonation data.

In reactive flow modeling using CHEETAH the EOS typically treats 20-30 species. Multiple chemical reactions can be used to treat the detonation kinetics, including reversible reactions. Fast reactions are assumed to be in equilibrium while slow reactions are treated directly. Chemical and density changes due to aging are easy to model by modifying the initial chemical compositions. In the ALE/CHEETAH chemically reacting flow model, the ALE code handles advection. Advection and chemical kinetics are time split. Calls are made by the ALE code to CHEETAH for EOS evaluations of the pressure, temperature and sound speed and for kinetic rate evaluations to update the species mass fractions. To update the species mass fractions the ALE code makes two rate calls to CHEETAH. The first takes a half time-step to obtain min-step values. These midstep values are then used by the ALE code to take a second order in time accurate full time-step. 
Internal to CHEETAH, the chemical kinetics is solved using an adjustable time step that sub-cycles within the fluid dynamic time step. CHEETAH allows the user to choose from several ordinary differential equation (ODE) solvers including efficient explicit 2nd and 4th order Runge-Kutta solvers and the high order implicit CVODE [5] ODE solver that can treat stiff kinetic rate equations.

Ordinary reactive flow models (e.g. Ignition \& Growth, JWL++, JTF [6--8]), use equations of state for only two species (reactant and product). For these models density changes are difficult due to model because of the very simple reactant EOS. The ALE/CHEETAH model treats multiple species and multiple (possibly reversible) chemical reactions in detonation. This leads to greater flexibility in modeling multiple non-equilibrium processes, such as those in composite explosives.

A key point of the ALE/CHEETAH

implementation that we will discuss in this paper is the use of a multi-dimensional sparse hash table in place of the direct calculation of EOS data. Table lookup and interpolation was found to be orders of magnitude faster than direct EOS evaluation, and a sparse table allows for a practical table size.

Rate equations in CHEETAH can take may forms:

- Pressure power-law

$$
\frac{d X_{i}}{d t}=A X_{i}^{B}\left(1-X_{i}\right)^{C} P^{D}
$$

- Arrhenius

$$
\frac{d X_{i}}{d t}=X_{i} X_{j} A T^{B} \exp \left(-\frac{E}{k T}\right)
$$

- Complex Chemkin (pressure, temperature) kinetic rate laws [9].

Reverse reactions can be calculated selfconsistently from the non-ideal EOS data, enforcing equilibrium species concentrations at large times.

\section{SPARSE HASH TABLE}

Dependent variables needed by the ALE model are calculated by CHEETAH using piecewiselinear, multi-linear or multi-dimensional polynomial interpolation from either a $2 \mathrm{D}(\rho, \mathrm{E})$ table or from a sparse hash table. New table entries are generated when needed by the interpolation scheme. For parallel calculations, sparse table entries are periodically passed to neighboring processors. Sparse table data is written to a binary file and can be input at the beginning of a calculation. Sparse or dense table data can be precalculated to improve efficiency and stability. The independent sparse table variables are $\left(\rho, E, X_{i}\right)$, which correspond to density, energy per gram, and mass fractions of the $\mathrm{N}$ species that are to be evolved using kinetic laws. There are $\mathrm{N}_{\mathrm{s}}=\mathrm{N}+2$ independent variables. Table spacing is fixed using either density or log density, energy, and molar concentration. The dependent variables stored in the table are $\left(\mathrm{P}, \mathrm{c}_{\mathrm{s}}, \mathrm{T}, \mathrm{c}_{\mathrm{V}}, \mathrm{X}_{\mathrm{j}}\right)$ which are the pressure, sound speed, temperature, heat capacity, and mass fractions of species in instantaneous equilibrium. Use of a sparse hash table was found to allow the strengths of the CHEETAH code to be applied to reactive flow modeling. Direct evaluation of the dependent variables is several orders of magnitudes slower than a table lookup. Dense tables become very large as the number of kinetic species increases. Sparse tables generated from trial calculations tend to correspond to data on a hyper-surface.

To calculate dependent variables it is first determined whether a fast 2D table or a sparse hash table should be used. The 2D tables can be used for un-reacted or fully reacted regions where the rate controlled species concentrations are fixed. 2D table lookup and interpolation is very much faster than for the sparse table. 2D fully reacted tables are only applicable to irreversible reactions.

In 2D and 3D ALE calculations both the number of spatial grid points and the number of time-steps can be very large. It is therefore important to consider the amount of work needed to evaluate data from the table look up. The 2D table is only used during EOS calls. The number of table evaluations needed per dependent variable for $2 \mathrm{D}$ tables scales as (Interpolation Order) ${ }^{2}$ per EOS call. For the sparse hash table the number of table lookups needed when using Multivariate Polynomial Interpolation scales as (Interpolation Order) $^{\mathrm{Ns}}$ per EOS call and as (Interpolation Order $)^{\mathrm{Ns}} \mathbf{x}(\mathrm{ODE}$ Order $)$ per rate call. The Multivariate Polynomial Interpolation scheme makes use of a product of polynomials that 
smoothly fit the data. This is very accurate if phase discontinuities do not exist, but can be very computationally intensive even for first order linear interpolation as the number of independent variables ${ }_{N s}$ increases. For linear interpolation and two kinetic controlled species the number of table evaluations needed for interpolation is $2^{4}$.

An alternative, faster interpolation scheme used by CHEETAH is Piecewise Linear Interpolation (PLI) [10]. For PLI the number of table lookups needed scales as $\mathrm{N}_{\mathrm{s}}+1$ per EOS call and as $\left(\mathrm{N}_{\mathrm{s}}+\right.$ 1)x (ODE Order) per rate call. The PLI scheme requires a sort of length $\mathrm{N}_{\mathrm{s}}$ that increases its operation count.

There are three ways that the Cheetah code can be used to do hydrodynamic kinetic modeling: a) directly calculation can be done for EOS and rate calculations at each call from the ALE code. (extremely slow); b) directly calculate data as needed to interpolate data and save the data at the interpolation points in 2D and sparse hash tables for future use. (slow initially as table builds); c) use a pre-build $2 \mathrm{D}$ and sparse hash tables to interpolate data. (fastest usage).

\section{RESULTS AND DISCUSSION}

We present the results from a $5 \mathrm{~mm}$ radius, $5 \mathrm{~cm}$ length rate-stick calculation for LX-17 (92.5\% TATB, 7.2\% KEL-F). We assume 2D cylindrical symmetry and have used a coarse mesh (40 zones per cm, 4000 zones total) for simplicity. The ratestick used was $5 \mathrm{~cm}$ length and impulse initiated. Calculations took roughly 1500 time-steps for the detonation wave to run the full length of the ratestick. Separate pressure dependent kinetic rates were used for TATB and KEL-F:

$$
\frac{d X_{i}}{d t}=A\left(1-X_{i}\right) P^{2},
$$

with pressure in $\mathrm{GPa}$, and $\mathrm{A}=0.0325 \mu \mathrm{sec}^{-1}$ for TATB and $0.02 \mu \mathrm{sec}^{-1}$ for KEL-F. The use of two kinetic rates leads to a four dimensional sparse table. We show in Fig. $1-4$ the spatial variation of several dominant species.

The number of table evaluations needed for a particular ALE/CHEETAH calculation is independent of the spacing of data points in the table. Unlike a dense table, decreasing the data spacing by a factor of 2 does not increase the number of sparse table entries by a factor of 2 for each dimension.

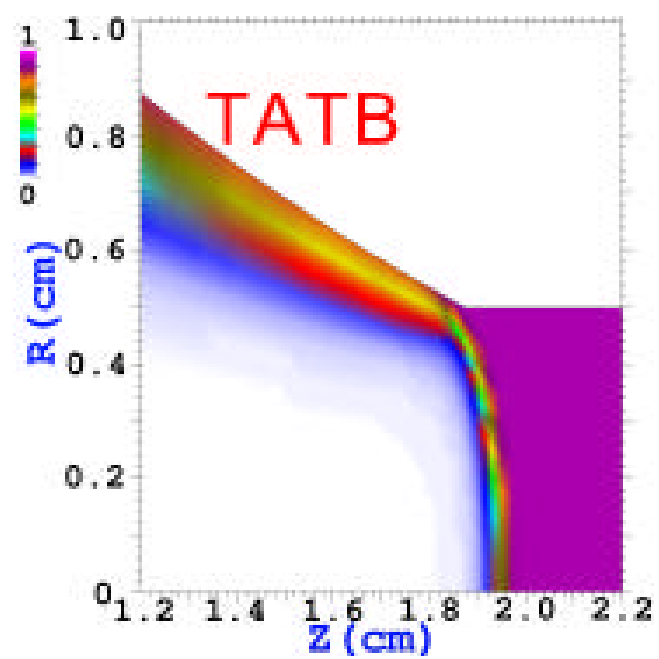

FIGURE 1. TATB mass fraction profile about the detonation front. The wave is moving from left to right.

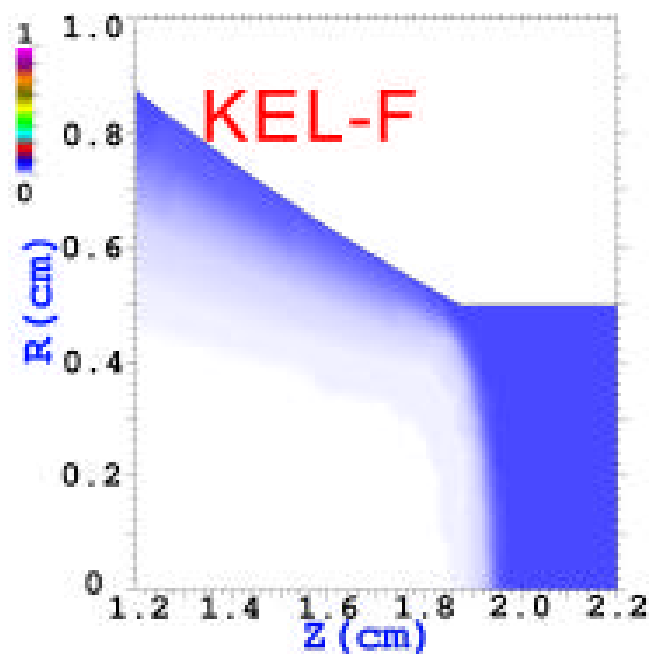

FigURE 2. KEL-F mass fraction profile.

Table 1

\begin{tabular}{|c|c|c|c|}
\hline $\begin{array}{c}\text { Table } \\
\text { Spacing }\end{array}$ & \begin{tabular}{c|} 
Number of \\
Table Entries
\end{tabular} & $\begin{array}{l}\text { Volume } \\
\text { of Table }\end{array}$ & $\begin{array}{l}\text { Number / } \\
\text { Volume }\end{array}$ \\
\hline $\begin{array}{l}\Delta \rho \quad 0.01 \mathrm{gm} / \mathrm{cc} \\
\Delta \mathbf{E} \quad 80 \mathrm{Cal} / \mathrm{gm} \\
\Delta \mathbf{X} 0.01 \\
\Delta X \quad 0.01\end{array}$ & 11,676 & 89,910 & 0.1313 \\
\hline $\begin{array}{l}\Delta \rho 0.005 \mathrm{gm} / \mathrm{cc} \\
\Delta \mathrm{E} \quad 40 \mathrm{Cal} / \mathrm{gm} \\
\Delta \mathbf{X} 0.005 \\
\Delta \times \mathbf{X} 0.005\end{array}$ & 44,060 & $1,104,000$ & 0.04245 \\
\hline
\end{tabular}


This is due to the hyper-surface nature of the sparse table (dimension $\sim 1.9$ for current problem). Table 1 shows that the sparseness decreases as the mesh spacing decreases. The volume is defined as the number of entries in an equivalent dense table.

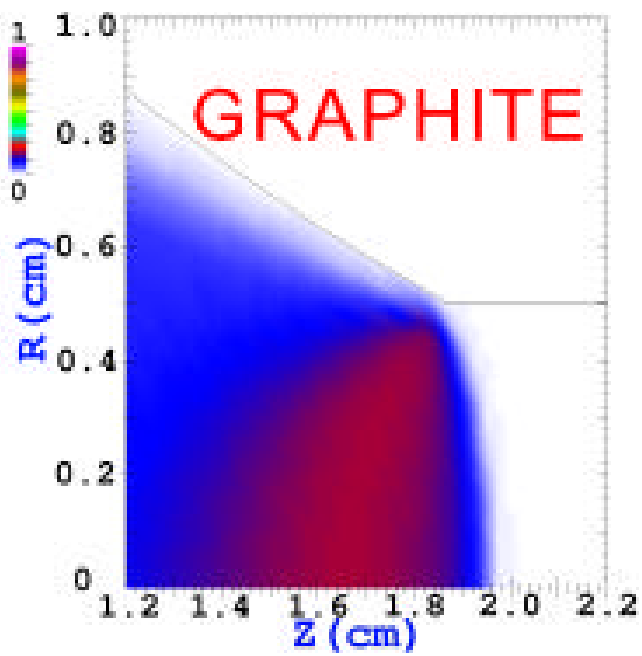

Figure 3. Graphite mass fraction profile.

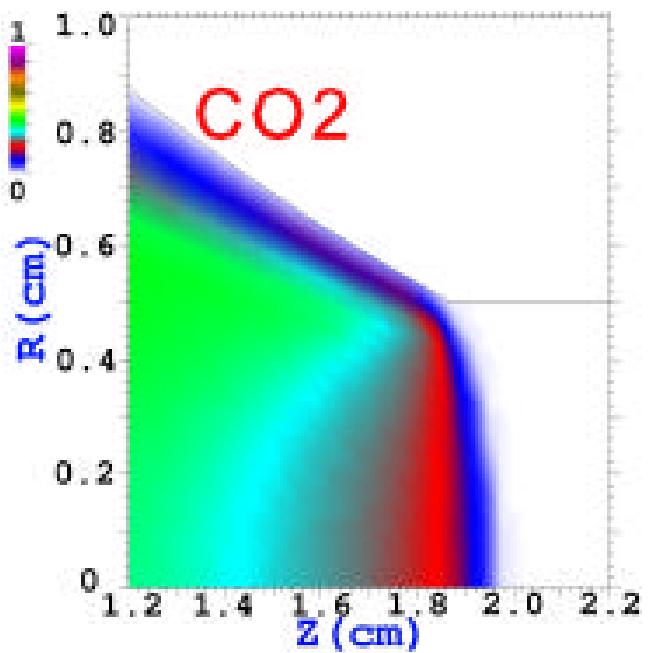

Figure 4. $\mathrm{CO} 2$ mass fraction profile.

Calculations of the detonation wave velocity were found to dependent very weakly upon: a) table data point spacing, b) ODE solver order, c) sparse table interpolation order or scheme. The timing was also similar for the 2 nd and 4 th order Runge-Kutta schemes, but was much longer ( 8 times) for CVODE solver. Timing was roughly $40 \%$ longer when sparse table spacing was halved.

\section{CONCLUSIONS}

ALE/CHEETAT is a new approach to reactive flow. The code is a flexible tool for a wide range of kinetic calculations. The model has been applied to small-scale and large-scale tests. Further work in numerical stability, user interface, and model development is still required

\section{ACKNOWLEDGEMENTS}

This work was performed under the auspices of the U.S. Department of Energy by the University of California Lawrence Livermore National Laboratory under contract No. W-7405-Eng-48.

\section{REFERENCES}

1. Fried, L. and Howard, M., "Cheetah 3.0 Users Manual", Lawrence Livermore National Laboratory UCRL-MA-117541, 2001.

2. Fried, L. E., and Howard, W. M., "An Accurate Equation of State for the Exponential-6 Fluid Applied to Dense Supercritical Nitrogen", J. Chem. Phys., Vol 109, p. 7338, 1998.

3. Murnaghan, F. D., Proc. Natl. Acad. Sci. (USA), Vol. 30, p. 244, 1944.

4. Souers, P. C., Forbes, J. W., Fried, L. E., Howard W. M., Anderson, S., Dawson, S., Vitello, P., and Garza, R., "'Detonation Energies from the Cylinder Test and CHEETAH v3.0,", Propellants, Explosives, Pyrotechnics, Vol 26, p. 180, 2001.

5. Cohen, S. D. and Hindmarsh, A. C., "CVODE User Guide," Lawrence Livermore National Laboratory Report UCRL-MA-118618, September 1994.

6. Lee, E. L. and Tarver, C. M., "Phenomenological Model of Shock Initiaiton in Heterogeneous Explosives," Phys. Fluids, Vol. 23, p. 2362, 1980.

7. Souers, P. C., Anderson, S., McGuire, E., and Vitello, P., "JWL++: A Simple Reactive Flow Code Package for Detonation," Propellants, Explosives, Pyrotechnics, Vol 25, p. 54, 2000.

8. Johnson, J. N., Tang, P. K., and Forest, C. A., "Shock-wave initiation of heterogeneous reactive shields,” J. Appl. Phys., Vol. 57, p. 4323, 1985.

9. Kee, R. J., Ripley, F. M., Meeks, E., and Miller, J. A., "CHEMKIN-III A Fortran Chemical Kinetics Package of the Analysis of Gas-Phase Chemical and Plasma Kinetics," UC-405 SAND96-8216, 1996.

10. Weiser, A. and Zarantonello, S. E., "A Note on Piecewise Linear and Multilinear Table Interpolation in Many Dimensions," Math. Comp., Vol 50, p. 189, 1988. 